\title{
THE STELLAR INITIAL MASS FUNCTION IN STARBURST GALAXIES
}

\author{
CLAUS LEITHERER \\ Space Telescope Science Institute \\ 3700 San Martin Drive, Baltimore, MD 21218
}

\begin{abstract}
Starburst galaxies are currently forming massive stars at prodigious rates. I discuss the star-formation histories and the shape of the initial mass function, with particular emphasis on the high- and on the low-mass end. The classical Salpeter IMF is consistent with constraints from observations of the most massive stars, irrespective of environmental properties. The situation at the low-mass end is less clear: direct star counts in nearby giant H II regions show stars down to $\sim 1 M_{\odot}$, whereas dynamical arguments in some starburst galaxies suggest a deficit of such stars.
\end{abstract}

\section{Introduction}

In this review I will define a starburst as a system in which high-mass stars dominate the energetics in terms of radiative and non-radiative heating. Stars falling under this definition have zero-age-main-sequence masses above $5-10 M_{\odot}$. Such a galaxy would be recognized as having blue colors, strong nebular emission-line spectrum, high inferred supernova rate, and (if dust is present) high IR luminosity. If less massive stars form down to about $1 M_{\odot}$ according to a Salpeter-like IMF, the gas consumption time-scale will be short in comparison with the Hubble time.

Three major starburst categories can be identified with this definition: IR-luminous galaxies, whose stellar population is enshrouded in dust (Sanders \& Mirabel 1996); nuclear starbursts, whose optical morphology is similar to that shown by Seyfert galaxies but the primary energy source being a young stellar population; starbursting dwarfs, whose optical spectrum is H II region-like, often with an indication of an older, underlying population (Gallagher 1996; Telles \& Terlevich 1997). 


\section{Techniques to derive the starburst IMF}

Massive stars are often embedded in gas and dust, which leads to absorption of stellar radiation and re-emission at longer wavelengths. On the one hand, this allows an analysis via nebular recombination lines of the stellar far-UV radiation, which would otherwise be inaccessible; but on the other, it means a significant fraction of UV light is absorbed, and heavily dust-obscured starbursts may not be visible at all. Techniques have been developed to analyze the directly emitted or reprocessed UV or far-UV light. These techniques are often rather indirect and make use of complex theoretical stellar models. "Observationally" derived IMFs in starbursts can sometimes be heavily dependent on such models. The willingness to believe in a variation or the universality of the IMF depends on the trust one has in stellar atmospheric and evolutionary models. The main diagnostics are:

Far-IR fluxes - Some or most of the stellar UV flux is used to heat dust grains, which reach an equilibrium temperature of about $40 \mathrm{~K}$. The associated far-IR emission peaks at about $60 \mu \mathrm{m}$ (Soifer et al. 1987). The agreement between star-formation rates in normal spiral galaxies derived from $\mathrm{H} \alpha$ and far-IR luminosities suggests that the far-IR luminosity traces massive stars (Devereux \& Young 1990).

Nebular emission lines - Nebular diagnostics are a major tool to study starburst populations. The subject has been reviewed by Stasinska (1996). The principle is to count photons, either from recombination lines or from excited transitions originating in the ISM, and then to infer the incident ionizing spectrum, which is then used to model the stellar population. The method is sensitive for stars with significant far-UV flux, i.e. temperatures above $30,000 \mathrm{~K}$, or masses above about $20 M_{\odot}$.

UV stellar-wind lines - The wavelength region between $912 \AA$ and $3000 \AA$ is dominated by stellar-wind lines. They result from absorption of photospheric far-UV photons, with an associated transfer of radiative into kinetic momentum. The profiles contain information on the stellar luminosity. Evolution models predict a stellar mass-luminosity relation so that the line profile is tied to the stellar mass (Mas-Hesse \& Kunth 1991; Robert, Leitherer, \& Heckman 1993; Leitherer, Robert, \& Heckman 1995).

Near-IR red supergiant features - The most prominent stellar absorption bands of cool stars are in the 1 to $2.5 \mu \mathrm{m}$ range (Lançon \& RoccaVolmerange 1992; Origlia, Moorwood, \& Oliva 1993). Evolutionary synthesis models for this spectral range (e.g., Rieke et al. 1993; Lançon \& Rocca-Volmerange 1996) provide constraints on the mass-to-light ratio (in combination with a dynamical mass) and on an additional diluting continuum due to non-thermal or dust emission. 


\section{IMF parameters}

The concept of the IMF was introduced by Salpeter (1955) in a study of the stellar content in the solar vicinity. It is worth recalling that the mass ranges sampled observationally in starbursts and in the solar neighborhood by Salpeter (1955) are not the same: Salpeter's result applies to stars with masses below $10 M_{\odot}$, whereas observations of starburst galaxies are usually restricted to higher stellar masses.

Excellent reviews have been written since Salpeter's pioneering work. I highlight Miller \& Scalo (1979), Tinsley (1980), and Scalo (1986). The IMF is often parameterized as a power-law $\phi(m) \propto m^{-\alpha}$, where $\phi$ is the number of stars formed per unit space and time. $\phi$ is defined in the interval $\left[M_{\text {low }}, M_{\text {up }}\right]$, where $M_{\text {low }}$ and $M_{\text {up }}$ are the lower and upper mass cut-offs, respectively. The slope $\alpha$ is 2.35 for a Salpeter IMF.

It is instructive to compare the starburst and the local field star IMF. The solar neighborhood IMF was derived by Miller \& Scalo (1979) and subsequently revised by Scalo (1986). A power law with an exponent of 2.3 is an acceptable fit to the Miller-Scalo IMF for the mass range above $10 M_{\odot}$. For the mass range above $10 M_{\odot}$, which is the one accessible for direct observations of starburst galaxies, the Scalo (1986) slope is consistent with a Salpeter slope of $\alpha=2.35$.

The mass range above $10 M_{\odot}$ is the tip of the iceberg. Only a few percent of the total stellar mass in a starburst is in massive stars. A large, uncertain extrapolation from $>10 M_{\odot}$ (the range from which stellar light is observed) to $1 M_{\odot}$ and below (where the mass is concentrated) is required to derive total star-formation rates and starburst masses.

Direct mass determinations can in principle be used to constrain the low-mass end of the IMF. Until very recently, obtaining stellar velocity dispersions in individual starburst clusters was technically infeasible. Massive stars which dominate optical and UV spectra have few suitable spectral lines. Furthermore, the velocities of their strong stellar winds by far exceed the expected velocity dispersions (about $10 \mathrm{~km} \mathrm{~s}^{-1}$ for a $10^{5} M_{\odot}$ starburst cluster). The most favorable conditions are in super star clusters where Ho \& Filippenko (1996) measured velocity dispersions, demonstrating the existence of stars with masses down to the $2-3 M_{\odot}$ range.

$M_{\text {up }}$ becomes statistically meaningful only for masses below $\sim 50 M_{\odot}$. Otherwise stochastic effects prevail since for typical starburst masses there are insufficient numbers of stars above about $50 M_{\odot}$ to contribute to the integrated light (Cerviño \& Mas-Hesse 1994). If $M_{\text {up }}$ is as low as $30 M_{\odot}$, the ionizing photon output of the population is cut drastically, and a soft ionizing radiation field is often ascribed to a low value of $M_{\mathrm{up}}$. 
To summarize the main points of this section, deriving the IMF in starburst galaxies is essentially focussed on three issues:

- determining the slope of the IMF above $\sim 10 M_{\odot}$;

- combining a mass and a light measurement to constrain $M_{\text {low }}$;

- measuring $M_{\mathrm{up}}$ from the hardness of the radiation field.

\section{Which stars form in different starburst types?}

\subsection{82 AND IR-LUMINOUS GALAXIES}

In their pioneering study, Rieke et al. (1980) introduced the terminology of the "top-heavy IMF". Top-heavy means a higher proportion of RSGs over red giants and dwarfs than expected for a normal IMF. In terms of mass, a top-heavy IMF has an excess of stars in the mass range $10-20 M_{\odot}$ over stars of $5 M_{\odot}$ and less. Observational evidence for a top-heavy IMF in M 82 comes from the relatively high K-band luminosity of the nucleus, together with its relatively low dynamical mass. Satyapal et al. $(1995 ; 1997)$ obtained spatially resolved near-IR spectroscopy of the central M 82 region. 12 unresolved starburst clusters contribute to the IR luminosity. They have an age dispersion of $6 \times 10^{6} \mathrm{yr}$ and suggest a propagation velocity of the starburst of $\sim 50 \mathrm{~km} \mathrm{~s}^{-1}$. Satyapal et al. (1997) find that a starburst model with a Salpeter IMF from 0.1 to $100 M_{\odot}$ fits the observational constraints of all clusters. It is interesting to note that starburst models can be found which do not require a deficit of low-mass stars.

M 82 is the low-luminosity extension $\left(L=3 \times 10^{10} L_{\odot}\right)$ of the class of IRluminous galaxies $\left(L>10^{11} L_{\odot}\right)$. IR-luminous galaxies are the dominant extragalactic population in the local universe, and most (if not all) undergo an intense starburst (Sanders 1997). Wright et al. (1988) determined $M / L$ ratios in a sample of interacting starburst galaxies. Unless most of the galaxy mass were concentrated in the starburst and the molecular gas were converted into stars at nearly $100 \%$ efficiency, the small $M / L$ ratios suggest a deficit of stars with masses $<5 M_{\odot}$. Detailed studies of individual objects like NGC 3256 (Doyon, Joseph, \& Wright 1994) point toward similar results.

A universal deficit of stars having masses $<5 M_{\odot}$ has important implications, such as a significant increase of the gas reservoir exhaustion timescale and the absence of a post-burst phase dominated by A stars.

The high-mass end of the IMF can be probed with nebular emission lines in the $\mathrm{K}$ band. IR-luminous galaxies are generally found to have a weak recombination spectrum, and therefore a soft stellar ionizing radiation field (Doyon, Puxley, \& Joseph 1992; Goldader et al. 1997). The most provocative interpretation would be a deficit of very massive stars, i.e., a steep or even truncated IMF above $30 M_{\odot}$. Alternative explanations are absorption of the ionizing photons by dust or mass-dependent dust extinc- 
tion. The latter scenario would imply that even in the $K$ band most of the massive stars are still buried in dust and not observable.

\subsection{H II GALAXIES}

The rich emission-line spectrum of H II galaxies allows the study of a key issue: the metallicity dependence of the IMF. Collisionally excited line ratios are sensitive to the electron temperature $T_{\mathrm{e}}$. Since the equilibrium temperature of the $\mathrm{H}$ II region depends on metallicity (which affects the cooling) and on the IMF (which controls the heating), the challenge is to disentangle IMF- and metallicity-induced variations of $T_{\mathrm{e}}$.

Stasińska \& Leitherer (1996) found that the emission-line spectra of 100 giant $\mathrm{H}$ II regions and starburst galaxies in the metallicity range $0.25 Z_{\odot}$ to $0.025 Z_{\odot}$ are consistent with being powered by a stellar population following a Salpeter IMF up to $\sim 100 M_{\odot}$. Low values of $M_{\text {up }}=30 M_{\odot}$ are not consistent with the data. At the metal-rich end, García-Vargas, Bressan, \& Díaz (1995a,b) applied an independent set of stellar and nebular models to a sample of giant H II regions with above-solar metallicity. As in the case of metal-poor systems, a Salpeter IMF without truncation at the upper end is consistent with the observations.

Direct signatures of ionizing stars, via UV absorption lines, have been detected in numerous starburst galaxies with IUE (Kinney et al. 1993). $H S T$ resolution (both spectrally and spatially) is required for quantitative IMF studies. Detailed analysis of the UV line profiles have been done, e.g., for NGC 1741 (Conti, Leitherer, \& Vacca 1996) and NGC 4214 (Leitherer et al. 1996). The strength and blueshift of C IV $\lambda 1550$ and Si IV $\lambda 1400$ require the presence of massive stars with masses of $50 M_{\odot}$ and higher. This is consistent with the two galaxies being classified as Wolf-Rayet galaxies (Conti 1991).

\subsection{ACTIVE GALACTIC NUCLEI}

The importance of starbursts in the AGN phenomenon has been emphasized repeatedly (e.g., Perry \& Dyson 1985; Cid-Fernandes \& Terlevich 1995). Regardless of what powers the AGN, it is now fairly well established that luminous starbursts can be found within tens of pc of the AGN (e.g., Colina et al. 1997) and that the nuclear starburst can make a significant contribution to the overall energetics of the AGN (Fanelli et al. 1997; Heckman et al. 1997).

Do the same stars form in the vicinity of an AGN as in typical starbursts? González-Delgado et al. (in prep.) obtained HST GHRS spectra covering the central 500 pc of the Seyfert2 galaxy NGC 7130 and compared them to a starburst region in the Wolf-Rayet galaxy NGC 1741. NGC 1741 
is a Magellanic-type irregular with quarter-solar metallicity. Its starburst is at the end of a central, kpc-size bar. In contrast, the (circum)-nuclear starburst in NGC 7130 has a chemical composition close to solar. Despite the different environment, the two spectra are virtually identical (except for the AGN emission-line contribution), suggesting rather similar stellar content. The strength of the absorption features in NGC 7130 excludes an additional source contributing to the starlight, such as a non-thermal continuum. Other observed galaxies in the sample (Mrk 477, NGC 5135, IC 3639) have very similar UV spectra, pointing toward similar massive-star content, and therefore similar IMF.

\section{The universality of the starburst IMF}

Is there a coherent picture emerging from the various pieces of evidence in different objects? In this section the main issues are summarized.

Temporal and spatial evolution - Star formation in starburst clusters, if they are spatially isolated, occurs nearly instantaneously. This is similar to the star-formation process in local high-mass star-formation regions where the observed age spreads are less than $2-3$ Myr (Massey et al. 1995a,b). There is increasing evidence for starbursts propagating on scales of tens of parsecs at velocities of $10^{1}$ to $10^{2} \mathrm{~km} \mathrm{~s}^{-1}$. Case studies are M 82 (Satyapal et al. 1995, 1997) or M 83 (Puxley, Doyon, \& Ward 1997). Starbursts appear to be confined to localized regions which can then trigger other starbursts in the vicinity. If observed at insufficient spatial resolution, propagating star formation will mimic a quasi-continuous starburst. On larger scales, starbursts in IR-luminous galaxies extending over hundreds of pc have age spreads which may be comparable to the burst ages themselves (tens of Myr). Otherwise the synchronization mechanism would be difficult to understand.

The shape of the IMF - Observations in different object classes generally lead to an IMF slope that is consistent with Salpeter's value $(\alpha=2.35)$. The IMF slope in starburst galaxies agrees within the error bars with the slopes derived in local regions of high-mass star formation. Massey et al. $(1995 \mathrm{a}, \mathrm{b})$ find a Salpeter IMF from number counts in the Galaxy, the LMC, and the SMC. The surveyed regions are Galactic open clusters and OB associations, and H II regions in the Magellanic Clouds, including 30 Doradus. Although not fulfilling the definition of a starburst, these regions are the closest local counterparts in existence. It is worth noting that most IMF determinations in starbursts refer to clusters. The field population of massive stars is yet unexplored and could well have a different IMF. Massey et al. derive a steeper IMF for massive field stars outside clusters in the Local Group of galaxies. 
The high-mass end - The masses of the most massive stars determined in dust-poor starbursts agree with those found in Local Group galaxies from an analysis of the Hertzsprung-Russell diagram. They are in the 50 to $100 M_{\odot}$ range. The stellar ionizing radiation field in dusty IRluminous galaxies is softer than expected for the IMF which is observed in dust-poor galaxies. It is unclear if this indicates a deficit of massive stars formed or observed. The first suggestion implies a truncation of the IMF, whereas the second an observational bias against detecting very massive stars, possibly due to obscuration effects. One might speculate that even at $2 \mu \mathrm{m}$ the most massive stars are not observable in IR-luminous galaxies.

The low-mass end - The low-mass end of the IMF (below $\sim 5 M_{\odot}$ ) in starburst galaxies is inferred from the observed $M / L$ ratio. Dynamical masses of starburst nuclei derived from rotation curves are relatively low, suggesting $M_{\text {low }} \approx 5 M_{\odot}$. However, observations of M 82 at high spatial resolution have revealed the morphological complexity of the region, making its interpretation in terms of starburst modeling a challenge. Velocity dispersion measurements in individual starburst clusters are an alternative method for a mass estimate. Results obtained for super star clusters in NGC 1569 and NGC 1705 suggest $M_{\text {low }}$ in the $1-3 M_{\odot}$ range. A priori there is no reason for the low-mass IMF in starburst galaxies and in local sites of massive-star formation to be the same. Nevertheless, local regions may give some guidance. HST has pushed the detection limit of stars in 30 Doradus and other regions to about $2 M_{\odot}$, with no indication for a truncation of the IMF (Hunter et al. 1995; 1997). Starburst galaxies and local regions show the same IMF at the high-mass end. Does this hold for the low-mass end as well?

The dependence on the environment - There is little indication for a significant influence of the environment, including the metallicity, on the IMF. Massive stars following a similar IMF form in the nuclei of starburst galaxies (e.g., NGC 7714), at the end of bars in irregular galaxies (e.g., NGC 1741), in dense super star clusters (e.g., NGC 1569), in candidate globular clusters in mergers (e.g., NGC 4038/39; Whitmore et al., in prep.), and around AGNs (e.g., NGC 7130). The derived IMF for the mass range $10-50 M_{\odot}$ is similar to that in local H II regions.

The universality of the IMF in starburst galaxies in the local universe is encouraging for efforts to understand the spectro-photometric properties of star-forming galaxies at high redshift. It may not be unreasonable to assume a similar IMF for the first generation of stars as well.

Acknowledgements - Travel support to attend IAU 186 from the LOC and from the AAS is gratefully acknowledged. 


\section{References}

Cerviño, M., \& Mas-Hesse, J. M. 1994, A\&A, 284, 749

Cid Fernandes, R., \& Terlevich, R. 1995, MNRAS, 272, 423

Colina, L., García-Vargas, M. L., Mas-Hesse, J. M., Alberdi, A., \& Krabbe, A. 1997, ApJ, 484, L41

Conti, P. S. 1991, ApJ, 377, 115

Conti, P. S., Leitherer, C., \& Vacca, W. D. 1996, ApJ, 461, L87

Devereux, N. A., \& Young, J. S. 1990, ApJ, 350, L25

Doyon, R., Joseph, R. D., \& Wright, G. S. 1994, ApJ, 421, 101

Doyon, R., Puxley, P. J., \& Joseph, R. D. 1992, ApJ, 397, 117

Fanelli, M. N., et al. 1997, AJ, 114, 575

Gallagher, J. S. 1996, in From Stars to Galaxies: The Impact of Stellar Physics on Galaxy Evolution, ed. C. Leitherer, et al. (San Francisco: ASP), 315

García-Vargas, M. L., Bressan, A., \& Díaz, A. I. 1995a, A\&AS, 112, 13

. 1995b, A\&AS, 112, 35

Goldader, J. D., Joseph, R. D., Doyon, R., \& Sanders, D. B. 1997, ApJ, 474, 104

Heckman, T. M., et al. 1997, ApJ, 482, 114

Ho, L. C., \& Filippenko, A. V. 1996, ApJ, 472, 600

Hunter, D. A., Light, R. M., Holtzman, J. A., Lynds, R., O’Neil, Jr., E. J., \& Grillmair, C. J. 1997, ApJ, 478, 124

Hunter, D. A., Shaya, E. J., Holtzman, J. A., Light, R. M., O'Neil, Jr., E. J., \& Lynds, R. 1995, ApJ, 448, 179

Kinney, A., Bohlin, R., Calzetti, D., Panagia, N., \& Wyse, R. 1993, ApJS, 86, 5

Lançon, A., \& Rocca-Volmerange, B. 1992, A\&AS, 96, 593

- 1996, New Astron., 1, 215

Leitherer, C., Robert, C., \& Heckman, T. M. 1995, ApJS, 99, 173

Leitherer, C., Vacca, W. D., Conti, P. S., Filippenko, A. V., Robert, C., \& Sargent, W. L. W. 1996, ApJ, 465, 717

Mas-Hesse, J. M., \& Kunth, D. 1991, A\&AS, 88, 399

Massey, P., Johnson, K. E., \& DeGioia-Eastwood, K. 1995a, ApJ, 454, 151

Massey, P., Lang, C. C., DeGioia-Eastwood, K., \& Garmany, C. D. 1995b, ApJ, 438, 188

Miller, G. E., \& Scalo, J. M. 1979, ApJS, 41, 513

Origlia, L., Moorwood, A. F. M., \& Oliva, E. 1993, A\&A, 280, 536

Perry, J. J., \& Dyson, J. E. 1985, MNRAS, 213, 665

Puxley, P. J., Doyon, R., \& Ward, M. J. 1997, ApJ, 476, 120

Rieke, G. H., Lebofsky, M. J., Thompson, R. I., Low, F. J., \& Tokunaga, A. T. 1980, ApJ, 238, 24

Rieke, G. H., Loken, K., Rieke, M. J., \& Tamblyn, P. 1993, ApJ, 412, 99

Robert, C., Leitherer, C., \& Heckman, T. M. 1993, ApJ, 418, 749

Salpeter, E. E. 1955, ApJ, 121, 161

Sanders, D. B. 1997, in Starburst Activity in Galaxies, ed. J. Franco, R. Terlevich, \& A. Serrano, Rev. Mex. Astron. Astrofis. Conf. Ser., 6, 42

Sanders, D. B., \& Mirabel, I. F. 1996, ARAA, 34, 749

Satyapal, S., et al. 1995, ApJ, 448, 611

Satyapal, S., et al. 1997, ApJ, 483, 148

Scalo, J. : 1986, Fund. Cosm. Phys., 11, 1

Soifer, B. T., et al. 1987, ApJ, 320, 238

Stasińska, G. is'J , in From Stars to Galaxies: The Impact of Stellar Physics on Galaxy Evolution ed. C. Leitherer, et al. (San Francisco: ASP), 232

Stasińska, G., \& Leitherer, C. 1996, ApJS, 107,427

Telles, E., \& Terlevich, R. 1997, MNRAS, 286, 183

Tinsley, B. M. 1980, Fund. Cosm. Phys., 5, 287

Wright, G. S., Joseph, R. D., Robertson, N. A., James, P. A., \& Meikle, W. P. S. 1988, MNRAS, 233, 1 